\title{
A Model Proposal for Long-Lasting Electromagnetic Forces-Biological System Interaction: Molecular Fatigue Damages
}

\section{Sert C}

\author{
Department of Biophysics, Medicine Faculty of Harran University, Turkey
}

${ }^{*}$ Corresponding author: Sert C, Department of Biophysics, Medicine Faculty of Harran University, Turkey, E-mail: csert@harran.edu.tr

Citation: Sert C (2016) A Model Proposal For Long-Lasting Electromagnetic Forces-Biological System Interaction: Molecular Fatigue Damages. J Biochem Biophy 1(1): 102

Received Date: January 26, 2016 Accepted Date: June 15,2016 Published Date: June 16, 2016

\begin{abstract}
None of the known interaction models of electric and magnetic fields with the biological system can completely explain the effects which occur as a result of long-lasting exposure to electric and magnetic fields.

We think that effects which cannot be explained by exposure to long-lasting, and albeit very low, electro-magnetic effects arise from molecular fatigue and the damage accumulation which occurs as a result of it.

Molecular fatigue is similar to the phenomenon which is observed in isotropic bodies (metals) and is defined as metal fatigue. Metal fatigue causes emergence of invisible capillary vessels in the material if loads and forces affect the material periodically by changing directions and if this effect is repeated many times. Fracture may occur even in stress values which are far below the tensile stress and shear stress, the reason behind this is molecular fatigue.

Molecular fatigue may emerge in anisotropic systems like bones and more complex systems in the human body such as cartilage, muscle much the same with isotropic bodies. Because biological system is a system which is exposed to constantly moving and repeating forces as well. These forces and gravitational forces act like a potential source by creating pressure electrification in the system and enable molecular production in these tissues. However, when the effects exceed certain values and durations, molecular fatigue occurs and brings about negative consequences in the live system with the emergence molecular fatigue damage accumulation in structures such as bones, muscles, cardiac muscles, receptors, ion channels.

Keywords: Long-lasting electromagnetic forces; Biological interaction; Molecular fatigue
\end{abstract}

\section{General Information}

Known interaction models of electric and magnetic fields with the biological system are as follows $[1,2]$

1 - Effects on cell surface structures.

2 - Polarization Forces.

3 - Coulombic Forces.

4 - Effects of the loads induced on cells.

5 - Surface loads induced on cell matrix and substrant.

6 - Cyclotron resonance and ion parametric resonance.

7 - Lednev model.

8 - Biologic electron transfer.

9 - Effects on free radical reactions.

None of interaction models can completely explain the effects which occur as a result of long-lasting exposure to electric and magnetic fields.

Many laboratory studies and epidemiological studies have reported that Electromagnetic fields cause serious health problems. One is exposed to electric and magnetic fields from high-tension lines, all kinds of electrical appliances used at homes and in industry, earth's magnetic field and medical devices such as Magnetic resonance [3].

In further in vitro experiments, Zhao, et al. found that application of physiological Direct current Electromagnetic field directed movement of bone cells and other cell types. This phenomenon is called electrotaxis or galvanotaxis [4]. 
EMF ( Electromagnetic field) frequencies in the body are normally in the range of extremely low frequencies (ELF). These EMF include the action potentials of nerves and heart tissue, skeletal muscle vibrations and frequencies elicited by rhythmic activities within other body tissues. Thus, we concentrate on these frequencies in the present review [5].

A current survey of the literature reveals many recent advances in our understanding of EMF, especially DC EF. Today we are experiencing a revolution because many cell biological findings are now explainable by ion dynamics $\left(\mathrm{Ca}^{2+}, \mathrm{H}^{+}\right.$and related ion pumps and voltage sensors) and their action on small signaling molecules. This clearly is a novel link to classical findings in molecular and cell biology. A number of insightful studies by groups including those of McCaig and Adams directly couple EMF to cell biology $[4,6,7]$. Levin demonstrated that DC EF produced by ion Channels (especially for $\mathrm{H}^{+}$, $\mathrm{K}^{+}$and $\mathrm{Ca}^{2+}$ ) provide specific signals that regulate cell behavior during embryonic development, normal tissue turnover and regenerative repair [8]. Thus far, roles have been proposed for endogenous currents (EF, ion currents and secondary distribution of small molecule in polarizing cells; patterning embryos during gastrulation, neurulation and organogenesis; directing transport of maternal components in insect oocytes; neural differentiation; guiding migration of many vertebrate cell types; Xenopus tail regeneration [9-13].

The most important candidate is ion movement (e.g. by voltage-sensitive $\mathrm{Ca}^{2+}$ channels) [14]. However, in the case of $\mathrm{K}^{+}$dependent signaling, $\mathrm{Ca}^{2+}$ fluxes were not affected by $\mathrm{K}^{+}$channel activity. Levin reports that in some studies, cell biological effects seem to depend on the particular transporter involved, indicating that the type of ion and perhaps its movement dynamics also act as signals $[8,15]$.

An additional effect for enhancing sensitivity (already addressed in the present review) was also discussed by Kindzelskii and Petty: the coherence and cooperative interaction of receptors to receptors or channels to channels (the distance of individual channels being only about $7 \mathrm{~nm}$ ) [16]. The coupling may take place via conformational mechanisms or via other coupling (electron tunneling or other quantum effects). All these mechanisms may further improve the sinyal amplification [16,17].

Berenice Noriega-Lunaa, et al, have seen that few studies report the influence of electromagnetic fields on cell structure [18]. Thus, the objective of this study was to evaluate the impact of a pulsed magnetic field of $0.70 \mathrm{mswidth}$, intensity of $0.65 \mathrm{mT}$ and frequency of $4 \mathrm{~Hz}$ on the structure of osteoblasts (cell line MG-63), in early stages of cell growth and in short periods of exposure. The systematic evaluation of this effect was performed by analysis of cell density, pattern of protein and microfibril distribution of F-actin and -tubulin and nuclear structure [18].

In recent studies, electromagnetic fields have been claimed to change dual interactions between nervous system, hormones and immune system. Their effect on DNA, RNA, protein synthesis, cell division, cell surface features, ion entry-exit from the cell membrane such as $\mathrm{Ca}, \mathrm{Na}$, ion bonding, signal transmission (neurotransmitters, hormones, enzymes), ion transport and activation of some enzymes have been researched [19-21]. With the impact of electromagnetic fields, atoms and molecules of the body may lose the electrical balance they established between each other, biochemical activities may get affected, electrical structure in the communication of cells and tissues may break down [22,23].

With ELF MF, immunity may deteriorate the impact on the functioning of immune system cells and tumor phenomena are claimed to become more frequent $[24,25]$. Other child cancers and mainly child leukemia, adult cancers, depression, suicide cases, cardiovascular diseases, reproduction disfunctions, developmental anomalies, immunological modifications, neurobehavioural effects and neurodegenerative diseases increase compared to normal population [26-28].

Long-lasting interaction consequences of ELF EMF can be evaluated under three main headings as cancer cases, cardiac results and diabetes according to experimental and epidemiological studies [26-29].

Average 2-2.25\% of child leukemias in the world have been reported to arise from electromagnetic fields at home. This values is twice the amount of the normal population30. Approximately 10-15\% of cancer cases have been found to be related to EM fields with 0-300 frequency [30].

Fields with $\mathrm{Hz}$ frequency exposed in everyday life have been demonstrated to increase brain tumors, leukemia and acute myeloid leukemia in men. Many epidemiological studies have demonstrated increased cancer incidence in residents living in the vicinity of high-tension lines or workers of these areas as well [31-35].

Savitz, et al, have researched the CV disease mortality related to occupational magnetic field in 140000 electricity workers. They have observed high mortality due to arrhythmia and acute myocardial infarction in workers who were exposed to high magnetic field for a longer period of time [36].

In the studies carried out in electricity workers in Sweden and Italy, it has been stated that low magnetic fields present no cardiovascular risk [37]. However, according to the findings of Savitz, deaths as a result of myocardial infarction and arrhythmia are possible with long-term $\mu \mathrm{T}$ level exposure [36].

In wide prospective cohort studies, it is accepted that heart disease will be increase in the next few years with change of HRV is [38-41]. 
In recent years, exposure to ELF EM fields has been accepted to trigger a certain diabetes type defined as Type III diabetes (Brittle Diabetes). According to this study, blood glucose level rises momentarily when approaching electromagnetic fields and returns to its normal values when withdrawn [42]. Nevertheless, Type II Diabetes may occur with very long-lasting exposure. According to this, one of the causes of ever-increasing Diabetes disease in the world population could be these fields. Such studies are being carried out.

We think that effects which cannot be explained by exposure to long-lasting, and albeit very low, electro-magnetic effects arise from molecular fatigue and the damage accumulation which occurs as a result of it.

Molecular fatigue: Molecular fatigue is similar to the phenomenon which is observed in isotropic bodies (metals) and is defined as metal fatigue [43]. Metal fatigue causes emergence of invisible capillary vessels in the material if loads and forces affect the material periodically by changing directions and if this effect is repeated many times [43]. Materials fatigue failure or damage has a molecular origin. It must be also taken into account that the mechanisms of damage under cyclic loading (fatigue conditions) may be different depending on material type (metals, ceramics, polymers, composites, biological tissue).

For this reason, the changes provoked by mechanical loading in the microstructure of materials (in particular, living tissues) need to be investigated.

As the periodic number and effect of the applied load increase, capillary cracks start to grow and material can sometimes fracture even as a result of a small force. Endurance of the strained material decreases with repeated cyclical load applications. Fracture may occur even in stress values which are far below the tensile stress and shear stress, the reason behind this is molecular fatigue $[43,44]$. Fatigue is a phenomenon which occurs not under static loads but dynamic loads $[43,44]$.

Molecular fatigue may emerge in anisotropic systems like bones and more complex systems in the human body such as cartilage, muscle much the same with isotropic bodies. Because biological system is a system which is exposed to constantly moving and repeating forces as well. These forces and gravitational forces act like a potential source by creating pressure electrification in the system and enables molecular production in these tissues $[45,46]$.

However, when the effects exceed certain values and durations, molecular fatigue occur and brings about negative consequences in the live system with the emergence molecular fatigue damage accumulation in structures such as bones, muscles, cardiac muscles, receptors, ion channels $[45,46]$.

Repetitive exposure to low density loadings for short periods causes low-level stress in the cortical bone and creates damage on microstructural level. However these repetitive motions in the bone tissure are of a potential stimulant nature in bone formation. Bone formation which occurs as in vivo may repair the damage which occurs in low-density loadings such as walking [45,46].

Muscle fatigue is defined as the loss of maximum strength and force output [47].

Recent studies have focused on understanding the fundamental mechanisms of muscle contraction and how partial fatigue factors distort contraction functions. Fatigue in running a marathon and other durability activities could be related to musle glycogen consumption, low blood glucose or dehydration $[47,48]$.

Cellular aspect of muscle fatigue is important. Factors which cause cellular muscle fatigue are considered as changes in stimulation contraction duality, ionic changes, distortions in cell metabolism, changes in cell ultrastructure. Out of these factors, stimulation contraction duality and changes in cell metabolism are most accentuated [47].

Many diseases are associated with ion entry and exit into cell. Ion flow occurs through ion channels. Ion channels are in the structure of macromolecular protein. These molecular structures work normal in their natural process, this work occurs by the interaction with another molecule. However, when these molecular structures are exposed to any external force long term as ELF EMF, their folding functions will be disrupted, cannot distribute the power falling on them and the molecule may lose its function in time. For example, muscle fatigue is known as increase in the time of $\mathrm{Ca}^{+2}$ transitions, Ca channels move slowly so relaxation time is extended [49-51]. Other reason of muscle fatigue is insufficient Na-K pump activity, and this is the pump protein [52]. As is seen, basis of fatigue at macro structures as muscle is the molecular fatigue and if it continues for a long time, dysfunctions may occur.

Diabetes, cancer and cardiac diseases depend on many reasons and they occur by long-term effects. Nowadays, there is great increase in the population of such diseases with growing environmental factors. Environmental ELF- EMF is one of these factors and these are available in many literatures given below.

In the increase of these diseases, by long-term exposure to ELF-EMF and functional deceleration and disorder by fatigue and occurrence of defects in molecule may possible. Piezzoelectrification in living bone tissue increases the collagen synthesis and bone tissue ranges normally, but extreme periodic loads cause molecular fatigue and capillary cracks in time [53]. ELF-EMF affects all cell membranes in general. Complex cell membranes detect and amplify poor electrical and chemical signals [54]. In order to make changes in cell, external EMF is not required to be penetrated into cell. By effecting signal transduction pathways, it may regulate cell proliferation and differentiation. ELF EMF can cause the initiation of disease process without being penetrated to cell membrane 
by anyway and even can provide transformation to a cancer phenotype [55]. Goldman, et al. showed that EMF effects Ca flow at cell membrane. In vitro experiments made in human tissues, they observed that ELF EMF had increased Ca flow [56]. Liburdy, et al. showed that ELF EMF had made it by opening CA channels rather than increasing Ca mobilization from endoplasmic reticulum [57]. Due to fact that it controls signal transduction, cell division, cell differentiation and cell proliferation, signal transmission exchange induced by EMF has a strong potential at tumor formation and promotion. Ca flow, which was induced by the exposition to EMF, causes the change and immunosuppression of T lymphocytes and other immune system factors. Changes in cellular Ca flow stimulate a group of enzyme which is called as protein kinases that play role in regulating various cell functions. Protein kinase activity is associated with cancer [58].

Cardiac activities completely depend on $\mathrm{Na}, \mathrm{K}, \mathrm{Ca}$ flow in cell membrane. It was shown in several studies that transition of these ions had been affected by EMP exposure. And it shows that channel proteins providing transmission of these ions were affected, and it is likely that cardiac diseases may occur by the formation of molecular fatigue in these molecular structures by long term EMF exposure [59]. However, cardiac diseases occur after the exposure of these areas for 5-6 years and cancer occurs after the exposure of these areas for 10-15 years [60]. So, it is difficult to make such long-term experimental studies and show molecular fatigue. However, according to current literature information, it is very likely that these diseases are associated with the molecular fatigue induced by EMF.

\section{Conclusion}

We think that similar to metal fatigue which occurs in isotropic bodies with constant dynamic forces, long-lasting electromagnetic effects cause disease and deaths by creating molecular fatigue and related damage accumulations in the live system.

\section{References}

1. Portier CJ, Wolfe MS (1998) Assessment of Health Effects from Exposure to Power-Line Frequency Electric and Magnetic fields. Working Group Rep 358-94.

2. Cifra M, Fields JZ, Farhadi A (2011) Electromagnetic cellular interactions, Prog Biophys Mol Bio 105: 223-46.

3. Magda H (2004) Biological Effects of Low Frequency Electromagnetic Fields. Environ Res Std 535.

4. Zhao M, Pu J, Forrester JV, McCaig CD (2002) Membrane lipids, EGF receptors, and intracellular signals colocalize and are polarized in epithelial cells moving directionally in a physiological electric field. Faseb J 16: 857-9.

5. Richard HW, Funk, Thomas Monsees, Nurdan Ozkucur (2009) Electromagnetic effects - From cell biology to medicine. Prog Histochem Cytochem 43: 177-264. 6. Zhao M, Song B, Pu J, Wada T, Reid B, et al. (2006) Electrical signals control wound healing through phosphatidylinositol-3-OH kinase-gamma and PTEN. Nature 442: 457-60.

7. Adams DS, Masi A, Levin M. (2007) $\mathrm{H}^{+}$pump-dependent changes in membrane voltage are an early mechanism necessary and sufficient to induce Xenopus tail regeneration. Development 134: 1323-35.

8. Levin M (2007) Large-scale biophysics: ion flows and regeneration. Trends Cell Biol 17: 261-70.

9. Robinson KR, Messerlie MA (2003) Left/right, up/down: the role of endogenous electrical fields as directional signals in development, repair and invasion. Bioessays 25: 759-66.

10. Woodruff RI (2005) Calmodulin transit via gap junctions is reduced in the absence of an electric field. J Insect Physiol 51: 843-52.

11. Uzman JA, Patil S, Uzgare AR, Sater AK (1998) The role of intracellular alkalinization in the establishment of anterior neural fate in Xenopus. Dev Biol 193: $10-20$.

12. Nishimura KY, Isseroff RR, Nuccitelli R (1996) Human keratinocytes migrate to the negative pole in direct current electric fields comparable to those measured in mammalian wounds. J Cell Sci 109: 199-207.

13. Adams DS, Masi A, Levin M. (2007) $\mathrm{H}^{+}$pump-dependent changes in membrane voltage are an early mechanism necessary and sufficient to induce Xenopus tail regeneration. Development 134: 1323-35.

14. Sasaki M, Gonzalez-Zulueta M, Huang H, Herring WJ, Ahn S, et al. (2000) Dynamic regulation of neuronal NO synthase transcription by calcium influx through a CREB family transcription factor-dependent mechanism. Proc Natl Acad Sci 97: 8617-22.

15. Ng CK, McAinsh MR (2003) Encoding specificity in plant calcium signalling: hot-spotting the ups and downs and waves. Ann Bot 92: 477-85.

16. Kindzelskii AL, Petty HR (2005) Ion channel clustering enhances weak electric field detection by neutrophils: apparent roles of SKF96365-sensitive cation channels and myeloperoxidase trafficking in cellular responses. Eur Biophys J 35: 1-26.

17. Neumann E, Siemens PM, Toensing K (2000) Electroporative fast pore-flickering of the annexin V-lipid sürfece complex, a novel gating concept for ion transport. Biophys Chem 86: 203-20.

18. Berenice NL, Myrna S, Modesto S, Mario AR (2011) Influence of pulsed magnetic fields on the morphology of bone cells in early stages of growth. Micron 42: 600-7.

19. Cleary SF (1993) A review of in vitro studies, low frequency electromagnetic filede. Am Ind Hyg Assoc J 54: 178-85.

20. Berg H (1999) Problems of weak electromagnetic field effect in cell biology. Bioelectrochem Bioenerg 48: 355-60.

21. Cridland NA, Sabour NR, Saunders RD (1999) Effect of $50 \mathrm{~Hz}$ magnetic field exposure on the rate of RNA synthesis by normal fibroblast. Int J Radiat Biol 75: 647-54.

22. Zusman I, Yaffe P, Pinus H, Ornay A (1990) Effects of pulsing electromagnetic filede on the prenatal and postnatal development in mice and rats in vivo and in vitro studies. Teratology 42: 157-70.

23. Pilla AA, Markov MS (1994) Bioeffects of weak electromagnetic filede. Rev Environ Health 10: 155-69.

24. Walleczek J (1992) Electromagnetic filede effect on cell of the immune system the role of calcium signalling. Faseb J 6: 3177-85. 
25. Trainor L (1993) Electromagnetic filede effect on people. Folia Med Cracov 34: 173-8.

26. Goodman R, Chismadzhev Y, Henderson A (1993) Electromagnetic filede and cells. J Cell Blochem 51: 436-41.

27. Jauchem JR (1990) Electromagnetic filede in there a danger. Lancet 6: 884.

28. Trappier A, Loria P, Johnson LP (1990) Evolving perspectives on the exposure risk from magnetic filede. J Natl Med Assoc 82: 621-4.

29. Pokorný J, Pokorný J, Kobilková J (2013) Integrative Biology Postulates on electromagnetic activity in biological systems and cancer. Integr Biol 5: 1439-46.

30. Kabuto M, Nitta H, Yamamoto S, Akiba S, Honda Y, et al. (2006) Childhood leukemia and magnetic fields in Japan: A case-control study of childhood leukemia and residential power-frequency magnetic fields in Japan. Int J Cancer 119: 643-50.

31. Sermage-Faure C, Demoury C, Rudant J, Clavel J, Hemon D, et al. (2013) Childhood leukaemia close to high-voltage power lines--the Geocap study, $2002-2007$. Br J Cancer 108: 1899-906.

32. Filho WV, Pelissari DM, Barbieri FE, Tone LG, Lee ML, et al. (2011) Exposure to magnetic fields and childhood acute lymphocytic leukemia in São Paulo, Brazil. Cancer Epidemiol 35: 534-9.

33. Linet MS, Hatch EE, Kleinerman RA, Kaune WT, Niwa S, et al. (1997) Residential exposure to magnetic fields and acute lymphoblastic leukemia in children. N Engl J Med 337: 1-7.

34. Kheifets L, Ahlbom A, Crespi CM, Roman E, Saito T, et al. (2010) A pooled analysis of extremely low-frequency magnetic fields and childhood brain tumors. Am J Epidemiol 172: 752-61.

35. Mezei G, Gadallah M, Kheifets L (2008) Residential magnetic field exposure and childhood brain cancer: A meta-analysis. Epidemiol 19: 424-30.

36. Savitz DA, Liao D, Sastre A, Kleckner RC, Kavet R (1999) Magnetic field exposure and cardiovascular disease mortality among electric utility workers. Am J Epidemiol 50: 1258-9.

37. Hakansson N, Gustavsson P, Sastre A, Floderus B (2003) Occupational exposure to extremely low-frequency magnetic fields and mortality from cardiovascular disease. Am J Epidemiol 158: 534-42.

38. Liao D, Cai JC, Rosamond WD, Heiss G, Barnes RW (1997) Cardiac autonomic function and incident coronary heart disease: A population-based case-cohort study. Am J Epidemiol 145: 696-706.

39. Graham C, Sastre A, Cook MR, Gerkovich MM (2000) Nocturnal magnetic field exposure: gender specific effects on heart rate variability and sleep. Clin Neurophysiol 111: 1936-41.

40. Martin TE, Coman CA, Shellock FG, Fair R, Pulling CC, et al. (2004) Magnetic resonance imaging and pacemaker safety at 1.5-Tesla. J Am Coll Cardiol 43: $1315-24$.

41. Dekker JM, Schouten EG, Klootwijk P, Pool J, Swenne CA, et al. (1997) Heart rate variability from short electrocardiographic ecordings predicts mortality from all causes in middle-aged and elderly men. Am J Epidemiol 145: 699-908.

42. Havas M (2008) Dirty electricity elevates blood sugar among electrically sensitive diabetics and may explain brittle diabetes. Electromagn biol med 27: 135-46 .

43. Suresh S (1998) Fatigue of materials. $2^{\text {nd }}$ ed, Cambridge press ISBN 0-521-57847-7.

44. Karenlampi PP, Pekka T, Pasi S (2003) Molecular fatigue in steamed wood. Int J Fatig 25: 489-97.

45. Weiner S, Wagner HD, Traub W (1999) The material bone: Structure-function relations. J Struc Biol 126: 241-55.

46. Martin RB (1991) Determinants of mechanical properties of bones. J Biomec 24: 79-88.

47. Fitts R (1996) Cellular, molecular, and metabolic basis of muscle fatigue. Handbook of physiology- section 12: 1151-83.

48. Miklo SZ, Steven BS, Carlos B, Henk L (2001) GranzierMechanical Fatigue in Repetitively Stretched Single Molecules of Titin. Biophys J 80: 852-63.

49. Allen DG, Lee JA, Westerbland H (1989) Westerbland. Intracellular calcium and tension during fatigue in isolated single muscle fibers from xenopus levis. J Phsiol 415: 433-58

50. Westerbland, Allen DG (1991) Chenges of myoplasmic calcium concentration during fatigue in single Mouse muscle fibers. J Gen Phys 98: 615-35.

51. Metzger JM, Moss RL (1987) Shortening velocity in skinned single muscle fibers. Biophys J 52: 127-31.

52. Sjøgaard G (1990) Execise-induced muscle fatigue: the significance of pottasium. Acta Physiol Scan Suppl 593: 1-63.

53. Lakes RS (1980) The role of gradient effects in the piezoelectricity of bone, IEEE T Bio-Med Eng 27: 282-3.

54. Miller EM (1999) Editorial by Technical editor of EMC Test and Design 6.

55. Non Thermal Effects of RF Electromagnetic Filede (1997) "Proceedings: International Seminar on Biological Effects of Non-Thermal Pulse and Amplitude Modulated RF Electromagnetic Fields and Related Health Risks ICNIRP 112.

56. Goodman EM, Greenebaum B, Marron MT (1995) Effects of electromagnetic fields on molecules and cells. Int Rev Cytol 158: $279-338$.

57. Liburdy RP (1992) Calcium signalling in lymphocytes and ELF fields: evidence for an electric field metric and a site of interaction involving the calcium ion channel. FEBS Lett 301: 53-9.

58. Litovitz KM, Krause D, Mullins JM (1991) Effect of coherence time of the applied magnetic field on ODC activity. Biochem Biophys Res Commun 178: 862-5.

59. Savitz DA, Liao D, Sastre A, Kleckner RC, Kavet R (1999) Magnetic field exposure and cardiovascular disease mortality among electric utility workers. Am J Epidemiol 50: 1258-9.

60. Sait ML, Wood AW, Sadafi HA (1999) A study of heart rate and heart rate variability in human subjects exposed to occupational levels of 50 Hz circularly polarised magnetic filede. Med Eng Phys 21: 361-9. 


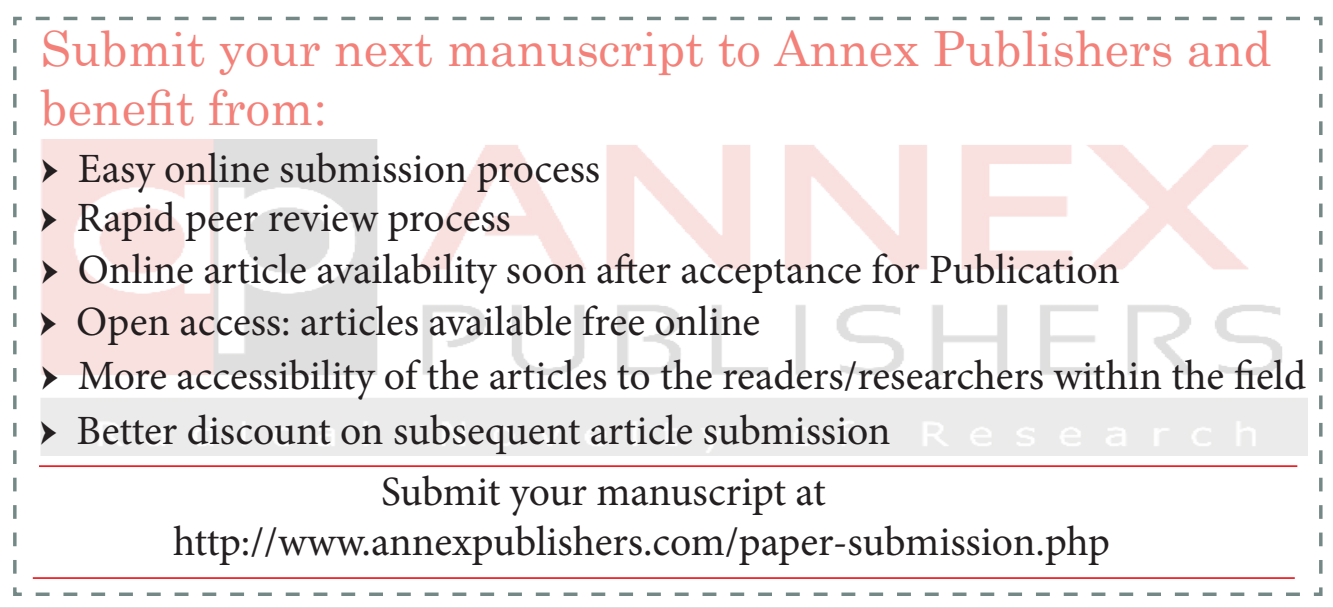

